\section{The Journal of \\ Laryngology \& Otology}

cambridge.org/jlo

\section{Online Only Abstract \\ Selection}

Cite this article: Abstracts presented at the South West ENT Academic Meeting 2018, Bath, UK. J Laryngol Otol 2019;E2:1-6. https:// doi.org/10.1017/S002221511900135X

First published online: 18 July 2019

\title{
Abstracts presented at the South West ENT Academic Meeting 2018, Bath, UK
}

Service provision prevents adequate training for core trainees in ENT

C Anderson ${ }^{1}$, D Osei-Kuffour ${ }^{2}$, S Rinkoff $^{2}$ and $\mathrm{H} \mathrm{Ubhi}^{3}$

From the ${ }^{1}$ Northwick Park Hospital, Harrow, ${ }^{2}$ Royal Free London NHS Foundation Trust and

${ }^{3}$ Epsom and St Helier University Hospitals

\section{Introduction}

The Joint Committee on Surgical Training state that core trainees in ENT 'should have the opportunity to attend three operating lists and three clinics per week'. It is often argued that service provision denies trainees these opportunities.

\section{Objective}

This study aimed to objectively measure whether ENT senior house officer rotas allow compliance with the Joint Committee on Surgical Training standards.

\section{Method}

Sixteen ENT core surgical training rotas were obtained by freedom of information requests. For each rota, the number of normal working days in 16 weeks - the average placement length - was calculated. It was anticipated that 3 days per week should be available for training; a total of 48 days per placement. It was assumed all normal days would be available for training, and that trainees would not be required to cover the wards or rota gaps.

\section{Results}

Two out of 16 placements (12.5 per cent) achieved 48 normal days in 16 weeks. In order to account for study leave (10 days) and annual leave (9 days), the number of weeks for achieving Joint Committee on Surgical Training indicators was lowered to 12, during which there should be at least 36 normal working days for training. Only four placements (25 per cent) had sufficient normal working days in a 12 -week period to allow Joint Committee on Surgical Training indicators to be met. This was due to the quantity of on-calls and rest days.

\section{Conclusion}

Conservative evaluation of this limited sample shows it is impossible for most core trainees in ENT to meet the Joint Committee on Surgical Training quality indicators due to service provision requirements, raising concerns about the experience of the ENT registrars of tomorrow.

Auditing a streamlined patient pathway for suspected vestibular schwannoma R Balakumar, H Schofield and S Gillett

From the Royal United Hospital, Bath

\section{Introduction}

The usual Royal United Hospital referral pathway for patients with unilateral sensorineural loss or tinnitus required at least two ENT out-patient visits, for assessment and for magnetic resonance imaging (MRI) results.

\section{Objective}

We hypothesised that a streamlined patient pathway would reduce appointment burden and patient travel time, and maintain safe diagnosis rates of vestibular schwannoma. 


\section{Method}

We performed a retrospective audit of audiology referral letters and their respective outcomes after the introduction of a streamlined patient pathway. Audiology referral letters were triaged by ENT specialists to: MRI where indicated, a follow-up hearing test, or no further action required. Negative MRI results were communicated by letter to the patient.

\section{Results}

A total of 156 referrals were audited. Of these, 66 per cent were triaged to imaging: 100 underwent MRI of the internal auditory meatus, and 3 underwent computed tomography because of contraindications. The incidence of vestibular schwannoma was 0.97 per cent ( 1 out of 103), and eight patients in total had abnormalities requiring further attention.

\section{Conclusion}

The streamlined referral pathway saved around 300 out-patient appointments over the audited period, and maintained a detection rate in line with the literature $(0.5-3$ per cent of patients scanned). Future improvements will look to increase audiology involvement in the screening process and review the standardised information provided to patients.

\section{Two years of bone conduction implant outcomes}

K Shanthakunalan, I Rothera, I Pai, R Obholzer, D Jiang and H R F Powell

From the Guy's and St Thomas' NHS Foundation Trust, London

\section{Introduction}

Bone-anchored hearing devices have been available in the UK since 1986. Developments in recent years include the avoidance of soft tissue reduction for percutaneous implants, the introduction of a punch technique and a drive towards transcutaneous devices. At St Thomas' Hearing Implant Centre, we offer a range of percutaneous and transcutaneous boneanchored hearing solutions.

\section{Objective}

Our aim was to review the outcomes of our patients receiving bone conduction implants in our Implant Centre over the last two years.

\section{Method}

All adult patients who had undergone bone conduction implant insertion between October 2015 and August 2017 were included in our study. A retrospective analysis of the database and each patient's notes was performed to collect data on: indication, type of surgery, post-operative wound infections and implant failures.

\section{Results}

Sixty-four patients were identified, of which 44 underwent minimally invasive Ponto surgery, and 20 had linear or curved incisions. The most commonly used abutment length was
$9 \mathrm{~mm}$. Eleven patients had post-operative wound infections that required oral antibiotic treatment, eight of whom had undergone a punch technique. The most common late complication was ongoing pain from the abutment site $(n=5)$, of which two patients went on to have the abutment removed. Implant failure occurred in five patients, three because of head trauma.

\section{Conclusion}

Our results suggest that minimally invasive Ponto surgery incurs more post-operative wound infections; however, it is proportionate when taking into account the ratio of procedures carried out using each technique. We believe there is a learning curve with the minimally invasive Ponto surgery technique. However, once overcome, this technique leads to comparable outcomes and less surgical time, which explains why some surgeons continue to perform minimally invasive Ponto surgery.

\section{A brief history of surgeons and their instruments: tonsillectomy}

\section{A Ashman}

From the Royal Berkshire Hospital/Oxford Deanery

\section{Introduction}

Learning about surgical history builds our understanding of our profession, and can often reveal some interesting stories and colourful characters.

\section{Objective}

The aim was to understand the historical development of a common surgical procedure, and thus give additional context to modern surgery.

\section{Method}

A review was conducted of the history of tonsillectomy as performed by the ancient Greeks, renaissance Europeans and more recent notable figures.

\section{Conclusion}

There is no such thing as a single, correct technique for tonsillectomy, and opinions are forever changing. Innovation in surgery is usually gradual and cumulative, with stepwise improvements being made by a series of individuals.

\section{Visual estimation of blood volume}

H R Davies, A S Harris and C D Passant

From the Aneurin Bevan University Health Board, Newport

\section{Introduction}

Otolaryngologists are often confronted with a patient who has bled from the upper aerodigestive tract, often following an operation such as tonsillectomy. Patients commonly estimate how much blood they have lost, but is this useful? 


\section{Objective}

This project aimed to assess how reliably people can estimate the volume of a blood sample using visual inspection only.

\section{Method}

This was a prospective study. Photographs were prepared of a standardised volume of artificial blood lying in a standard bathroom sink. Three photographs were prepared of three different volumes $(50 \mathrm{ml}, 250 \mathrm{ml}$ and $500 \mathrm{ml})$. Participants were hospital staff selected at random. Each participant was shown each of the three photographs three times in a randomised order and asked to estimate the quantity (in millilitres) of blood shown.

\section{Results}

Fifty-three participants were included ( 22 doctors, 21 nurses and 10 allied health professionals). In order to facilitate comparison, an 'error factor' (= estimated volume / actual volume) was utilised. Mean estimates of quantity were: $79.48 \mathrm{ml}$ for 50 $\mathrm{ml}$ blood volume (error factor of $1.59 \pm 1.40$ ); $178.13 \mathrm{ml}$ for $250 \mathrm{ml}$ blood volume (error factor of $0.71 \pm 0.62$ ); and $286.44 \mathrm{ml}$ for $500 \mathrm{ml}$ blood volume (error factor of $0.57 \pm$ 0.46). The overall mean error factor standard deviations by profession were: doctors $=0.654$, nurses $=1.032$ and allied health professionals $=0.667$.

\section{Conclusion}

This study showed that participants were generally inaccurate in visually estimating blood volume. The error factors show that participants tended to overestimate smaller volumes and underestimate larger volumes. Clinicians should exercise caution when told an estimated blood loss by their patients, as even experienced hospital staff members are very inaccurate at doing this.

A survey to assess the productiveness of text message reminders as a resourceful tool to improve clinic attendance rate

D Nair and S Pal

From the Ealing Hospital, London

\section{Introduction}

Text messaging to remind patients of their clinic appointments has been in practice across National Health Service trusts. We agree with the concept, but contest that the quality of its content is imperfect.

\section{Objective}

We aimed to demonstrate that the contents of the text reminders should be exhaustive, and include the name of the consultant and department, in addition to the standard information relating to date, time and hospital.

\section{Method}

A questionnaire was constructed to assess the current information in the text reminders received by patients who attended our department over a two-week period. It was distributed to patients on arrival, in the ENT department.

\section{Results}

We collected responses from 65 patients, of which 34 (52.30 per cent) were new and 31 (47.70 per cent) were attending follow-up appointments. Only 14 (41.17 per cent) of new and 15 (48.38 per cent) of follow-up patients received a text reminder; all of them received it within 72 hours of the appointment date. A large majority (65.51 per cent) responded, stating a lack of information about the department or consultant they were seeing. Six patients (20.68 per cent) referred to their appointment letter to obtain relevant information. All responders went to the main out-patients reception first, to confirm their appointment. Of the patients, 66.15 per cent (43 out of 65) expressed their preference to receive both a letter and text reminder for future appointments.

\section{Conclusion}

The text reminder service is a useful tool to improve clinic attendance rates. Currently, the contents of the text reminder are incomplete. The authors are in discussion to improve the service for better system performance.

Dizziness and driving - what are the rules and are we advising patients correctly? A completed cycle audit

J Sinnott, H Mahoney, A Wilkinson and S Broomfield

From the University Hospitals Bristol

\section{Introduction}

Safety surrounding driving is incredibly important and it is imperative that clinicians are giving appropriate advice. Patients suffering with vertigo are commonly encountered within ENT. The Driver and Vehicle Licensing Agency ('DVLA') has comprehensive guidance for patients with dizziness. However, these rules leave considerable scope for interpretation by clinicians. The Driver and Vehicle Licensing Agency medical advisors and Medical Defence Union advisors were contacted for clarification. It is the patient's responsibility to inform the Driver and Vehicle Licensing Agency of their condition and it is the clinician's role to advise the patient. However, many previous studies have shown that ENT doctors have poor knowledge of driving legislation.

\section{Objectives}

We aimed to assess the advice given to patients in a vertigo clinic regarding driving, and to design and produce a patient information leaflet.

\section{Method}

An audit was performed of driving advice given to 100 patients referred for further vestibular testing. A patient information leaflet was produced and teaching sessions provided; a re-audit of 50 patients was then performed.

\section{Results}

Only 6 of the 100 patients audited in the first cycle received any driving advice. After leaflet production and teaching 
sessions, 5 out of 50 patients were given driving advice; this equates to an increase from 6 per cent to 10 per cent in patients receiving advice.

\section{Conclusion}

There are multiple likely reasons for the deviation from the Driver and Vehicle Licensing Agency guidelines. This presentation aimed to clarify the current guidelines on when we should be advising patients to inform the Driver and Vehicle Licensing Agency, present the leaflet created and to make recommendations for best future practice.

\section{A citation analysis of the top 50 papers in ENT literature}

\section{$\mathrm{N}$ Jones $^{1}$ and R Ologunde ${ }^{2}$}

From the ${ }^{1}$ College of Medical and Dental Sciences, University of Birmingham and ${ }^{2}$ Institute of Head and Neck Studies and Education, University of Birmingham

\section{Introduction}

The surgical specialty strives to base its clinical practice on evidence-based medicine. However, the current evidence base in many surgical subspecialties remains lacking. This study aimed to identify the most cited papers in the ENT surgery literature and perform a citation analysis identifying the evidence levels of the studies.

\section{Method}

The authors identified the 50 most cited articles published in the 20 highest impact factor (as of January 2018) ENT surgery journals, through the Web of Science. The articles were ranked in order of number of citations acquired, and the levels of evidence were determined.

\section{Results}

The top 50 cited papers were published in 12 different journals between the years 1982 and 2014. The average level of evidence was 3.34. The majority of papers $(n=22)$ were of level 5 evidence. Nine articles were of level 1 evidence, and these consisted mainly of position papers and guidelines.

\section{Conclusion}

In the ENT surgical literature, there is no positive correlation between a high number of citations and evidence level. The majority of highly cited papers are level 5 evidence scientific research papers. With a drive towards higher levels of evidence to guide clinical management, newer papers of higher levels of evidence are becoming cited increasingly more often.

\section{Orbital infection: a complete cycle seven-year audit} and a management guideline

\section{Atfeh ${ }^{1}$, K Singh $^{2}$ and H S Khalil ${ }^{1}$}

From the ${ }^{1}$ Plymouth University, Peninsula Schools of Medicine and Dentistry and ${ }^{2}$ King's College Hospital, London

\section{Introduction}

This article presents a seven-year complete cycle audit project that aimed to systematically evaluate and standardise our clinical practice regarding the management of orbital infections.

\section{Objective}

Orbital infections are regularly encountered and are managed by various healthcare disciplines. Sepsis of the orbit and adjacent tissues can be associated with considerable acute complications and long-term sequelae. Therefore, prompt recognition and management of this condition are crucial. This article presents the outcomes of a seven-year complete cycle audit project and describes the development of the new local guideline on the management of orbital infections in our tertiary centre.

\section{Methods}

The study involved: (1) a retrospective five-year audit cycle on patients with orbital infections; (2) a review of available evidence on the management of orbital infections; (3) a new local multidisciplinary guideline on the management of orbital infections; and (4) a retrospective two-year second audit cycle to assess the clinical outcomes.

\section{Results}

Various disciplines intersect in the management of orbital infections. Standardising the management of this condition proved to be achievable through the developed guideline. However, room for improvement in practice exists in some areas, such as the promptness in referring patients to specialist care, the multidisciplinary assessment of patients on admission, and the improvement in patients' scanning requests.

\section{Conclusion}

There has been an observable improvement in local practice associated with the implementation of the constructed guideline on the management of orbital infection. However, further steps are required in order to develop our care of orbital infections patients.

\section{Development of a novel scoring system to gain} prognostic information using gallium scanning in necrotising otitis externa

\section{S Hollis, P Clamp, J Kabala and L Armstrong \\ From the University Hospitals Bristol NHS Foundation Trust}

\section{Introduction}

We wanted to help guide the duration of costly and inconvenient antimicrobial therapy in necrotising otitis externa cases by devising a scan scoring system to quantify the response to treatment.

\section{Objective}

We aimed to assess the correlation of gallium scan scores with clinical information, to guide prognosis and provide a diagnosis of necrotising otitis externa. 


\section{Method}

A retrospective analysis was conducted of gallium scan scores, patient co-morbidities and clinical findings at the time of each scan. Scan scores were generated by assessing the uptake signal in the bone marrow of both temporal bones, and comparing them to a score from a normal bone marrow signal from the $\mathrm{C} 4$ vertebral body. This resulted in a ratio of signal relative to the affected side (if only unilateral disease). These scores were then analysed against a range of clinical parameters.

\section{Results}

Sixteen of 36 scans had a ratio result of greater than 1.347 (defined as abnormal in this study). In this group, a greater proportion of patients had otalgia (80 per cent vs 60 per cent), cranial nerve palsy (44 per cent vs 27 per cent) and skull base involvement ( 56 per cent $v s 7$ per cent), with significantly higher C-reactive protein levels and prognostic scores in this group. Mortality was also increased in the abnormal scan result group (50 per cent vs 27 per cent).

\section{Conclusion}

This study demonstrates a tendency for significance between abnormal scan ratio results and markers of necrotising otitis externa morbidity and mortality. Current numbers are too small for statistical significance; further data are currently being collected.

\section{Availability of assistive devices for hearing loss patients in audiology and ENT clinic reception areas: results from a cross-sectional survey in England}

\author{
G M Jama ${ }^{1}$, S Shahidi ${ }^{2}$, J Danino ${ }^{1}$ and J Murphy ${ }^{1}$ \\ From the ${ }^{1}$ Royal Wolverhampton NHS Trust and ${ }^{2}$ University \\ Hospitals Birmingham NHS Foundation Trust
}

\section{Introduction}

Fair and equal access to healthcare for all is a fundamental principle of the National Health Service (NHS). Under the Equality Act 2010, healthcare providers in the UK are required, by law, to ensure that their services are accessible to people with disabilities and sensory loss, including those with hearing loss. The evidence shows, however, that hearing-impaired patients are more likely to suffer ill health compared to the rest of the population, largely as a result of avoidable communication barriers when using health services.

\section{Objective}

This study aimed to investigate the extent to which assistive technologies for patients with hearing loss, such as induction loop systems and visual alert displays, are available at reception desks, and in patient waiting areas in audiology and ENT outpatient clinics in England.

\section{Method}

A cross-sectional, confidential telephone survey of all NHS trusts in England providing audiology and ENT services was conducted. A structured questionnaire was administered to receptionists at selected out-patient departments.

\section{Results}

A total of 208 receptionists were contacted, of which 208 agreed to participate. Assistive technologies were available at the waiting areas in 64 per cent of audiology clinics (49 out of 76) and 42 per cent of ENT clinics (32 out of 76), and in 71 per cent of shared audiology and ENT reception areas (40 out of 56).

\section{Conclusion}

The availability of assistive devices varies between audiology and ENT clinic reception areas. Overall, there is a need for greater provision of appropriate communication support, to ensure that out-patient services become more accessible to patients with hearing loss.

\section{Re-evaluating how we manage epistaxis: taking a multidisciplinary approach to designing and implementing a new epistaxis pathway at a UK teaching hospital}

A Liu, P Schorscher, Z Osmani, E Crossley and K Desai

From the Brighton and Sussex University Hospitals Trust, Brighton and Sussex Medical School

\section{Introduction}

Epistaxis management continues to vary across the UK. Some units discharge low-risk patients with nasal packs in situ, for out-patient review 24 hours later. In light of continuing hospital pressures and new British Rhinological Society guidelines, is this approach safe and justifiable?

\section{Objectives}

We aimed to: evaluate current epistaxis management at a teaching hospital through audit; design and implement a new epistaxis management pathway; and predict the impact of new pathway.

\section{Method}

Data were retrospectively collected from all patients who presented to the accident and emergency department (A\&E) with epistaxis from January to April 2014. A consensus meeting was held between the A\&E, acute ambulatory care and ENT departments to agree a new Trust epistaxis pathway. Further data were collected between January and December 2014 to project likely hospital bed savings.

\section{Results}

A total of 146 patients were seen from January to April 2014, with a mean age of 61 years. Nasal cautery was performed in 4.4 per cent of patients $(n=16)$. Nasal packing was received by 31.5 per cent of patients $(n=46)$, of which 50 per cent $(n=23)$ required bilateral packing and 6.5 per cent $(n=3)$ required subsequent sphenopalatine artery ligation. Antithrombotic 
medication was administered to 8.2 per cent of patients $(n=12)$, all of whom were admitted.

In total, 32.1 per cent of patients $(n=47)$ required admission, and the average length of stay was 3 days. The length of stay for patients with bilateral versus single packs was significantly longer (3.7 vs 2.6 days, $p=0.04$ ). Over the entire year, 365 patients were seen and 29.0 per cent $(n=106)$ were admitted. Of these, 26.4 per cent $(n=28)$ were eligible for discharge on the new pathway, saving 78.4 hospital bed days. No patients identified as low risk re-bled or were re-admitted.

\section{Conclusion}

Whilst the number of patients eligible is small, there is significant potential for cost savings, whilst continuing to manage patients safely.

\section{Streamlining tonsillitis and peritonsillar abscess treatment: the new 4-hour target}

T Williams, E Heywood, M Saad and G Watson

From the ENT Department, Royal Hallamshire Hospital, Sheffield Teaching Hospitals

\section{Introduction}

There has been a significant rise in tonsillitis and peritonsillar abscess related admissions and complications. This can be attributable to a substantial reduction in tonsillectomies since the introduction of the 'procedures of limited clinical effectiveness' policy. As a result, there has been an increasing amount of hospital bed days associated with these common ENT presentations, with significant financial burden.

\section{Objective}

We aimed to create, implement and evaluate an evidencebased, 4-hour treatment bundle for the management of tonsillitis and peritonsillar abscess.

\section{Method}

All patients who presented to a large teaching hospital with tonsillitis or peritonsillar abscess over two 1-month periods were included, between which the 4-hour treatment bundle was implemented. This treatment included the use of intravenous fluids, analgesics, antibiotics and corticosteroids, plus needle aspiration in peritonsillar abscess patients. Outcomes measured were overnight admission and re-admission rates.

\section{Results}

Admission rates reduced from 75 per cent to 25 per cent after the introduction of the treatment bundle. There were no re-admissions after introduction of the new treatment bundle, compared with three re-admissions in the month prior.

\section{Conclusion}

The implantation of a 4-hour treatment bundle safely reduces the admission rates of patients with tonsillitis and peritonsillar abscess. These data are being used to introduce an ambulatory day unit on the ward, to further provide a streamlined, safe treatment service to reduce pressure on beds.

\section{SerenoCem granules: Medicines and Healthcare} products Regulatory Agency product alert and audit of patients

\author{
J Sinnott and P Clamp \\ From the University Hospitals Bristol
}

\section{Introduction}

In August 2017, a national Medicines and Healthcare products Regulatory Agency ('MHRA') medical device alert was issued for SerenoCem. This product, consisting of small granules made from porous cement, has been used in the obliteration of mastoid cavities, and there was new evidence of possible bone resorption. All patients implanted with SerenoCem needed to be identified and clinically reviewed. A major British Society of Otology audit was initiated. We describe our local experience of the audit and the results of the patients from our centre.

\section{Objective}

We aimed to identify and clinically review all patients implanted with SerenoCem granules. We analysed all imaging studies, symptoms and clinical examination findings, and reviewed the operation notes of any revision cases to look for evidence of erosion.

\section{Method}

Patients were identified using a search of keywords in the electronic notes and patient records. All patients were written to, offering them an out-patient appointment. Imaging studies, out-patient letters and operation notes were reviewed.

\section{Results}

In Bristol, between 2003 and 2011, 34 patients were implanted with SerenoCem granules. Patients' ages ranged from 15 to 80 years. There was evidence of bone erosion in some symptomatic patients, but no complications such as facial nerve palsy. Operative removal of the granules usually resulted in a dry, infection-free cavity, and the elimination of inflammation.

\section{Conclusion}

There was evidence of bone erosion in some symptomatic patients. Removal of the granules seemed to resolve symptoms. It remains unclear whether there is the possibility of erosion in asymptomatic patients, but there was no evidence of this in the small number of patients reviewed. 